\title{
Supplimentary Information
}

\section{Growth of Colloidal Nanoplate Liquid Crystals Using Temperature Gradients}

Abhijeet Shinde, ${ }^{a, \ddagger}$ Dali Huang,,${ }^{b} \neq$ Mariela Saldivar, ${ }^{a}$ Hongfei Xu, ${ }^{a}$ Minxiang Zeng, ${ }^{a}$ Ugochukwu Okeibunor, ${ }^{a}$ Ling Wang, ${ }^{a}$ Carlos Mejia, ${ }^{a}$ Padetha Tin, ${ }^{c}$ Sasha George, ${ }^{a}$ Lecheng Zhang a and Zhengdong Cheng $* a, b, d$

a. Artie McFerrin Department of Chemical Engineering, Texas A\&M University, College Station, Texas 77843, United States

b. Department of Materials Science and Engineering, Texas A\&M University, College Station, Texas 77843, United States

c. NASA Glenn Research Center, Cleveland, Ohio 44135, United States

d. Professional Program in Biotechnology, Texas A\&M University, College Station, Texas

77843, United States

${ }^{*}$ Corresponding author

${ }^{\ddagger}$ Authors contributed equally to this work

Address for correspondence: zcheng@tamu.edu

(a)

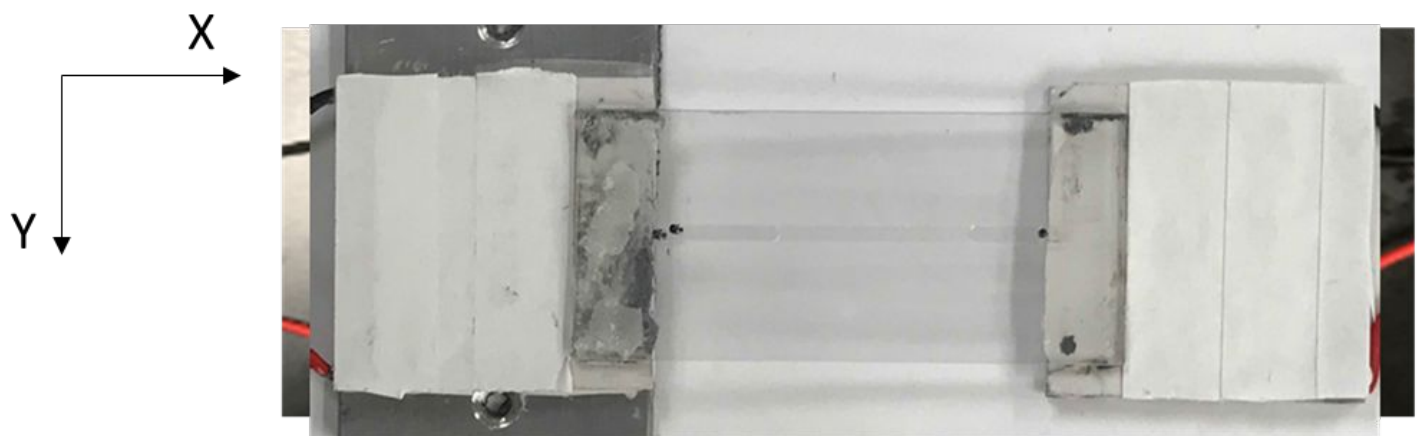

(b)

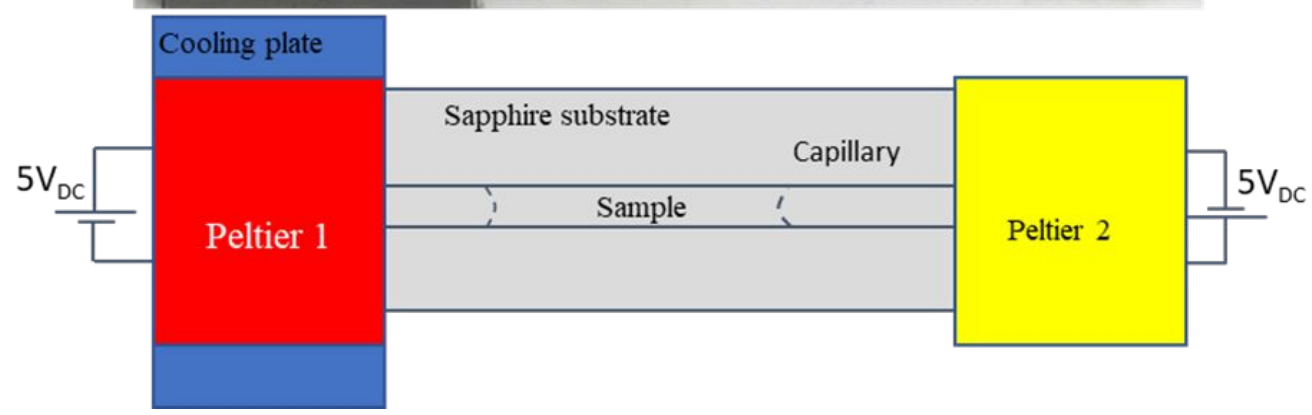

Figure S1. (a) Image of the capillary, containing the sample, placed on the transparent sapphire crystal plate that had its ends in contact with the Peltier devices. The left end was in contact with the cold surface of the Peltier device and a cooling plate was used to take away heat from the hot surface of the Peltier device. The 
right end of the sapphire plate was in contact with the hot surface of the Peltier device. The voltage supply units, fan used for the cooling plate are not shown in the image. (b) Schematic of the thermal gradient setup. The setup was placed in $\mathrm{XY}$ plane with $\mathrm{X}$-direction pointing towards horizontal direction of increasing temperature and $\mathrm{Y}$-direction was along gravity.

(a)

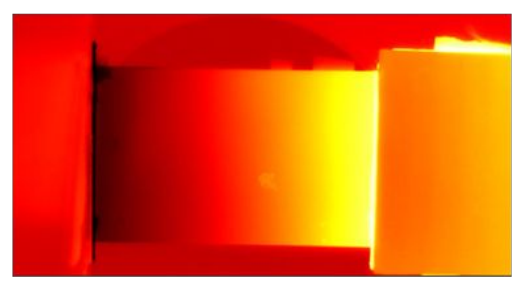

(b)

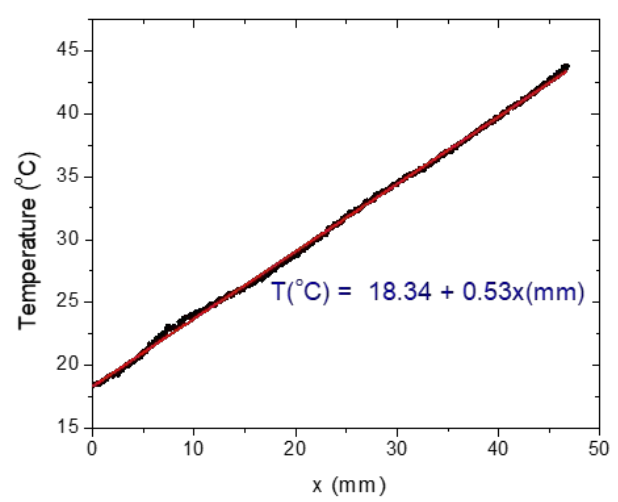

Figure S2. (a) FLIR image of the temperature gradient substrate obtained 5 minutes after turning on the Peltier devices (b) Linear temperature profile along the horizontal (x) direction measured from part a.

(a)

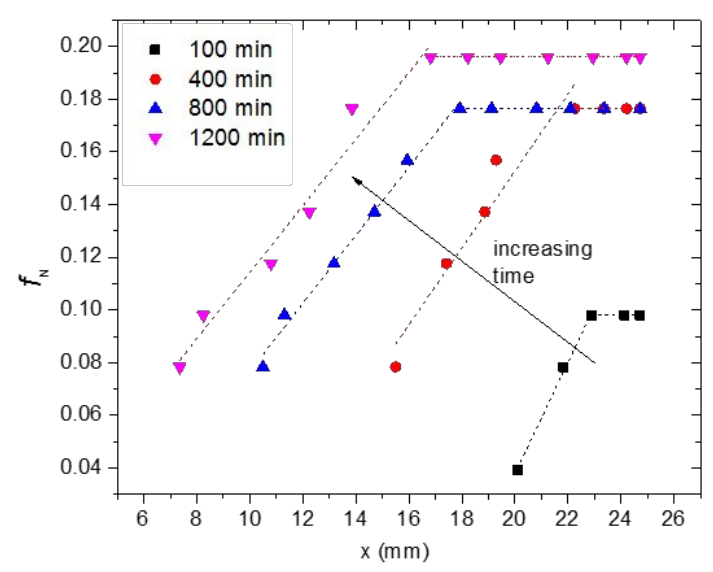

(b)

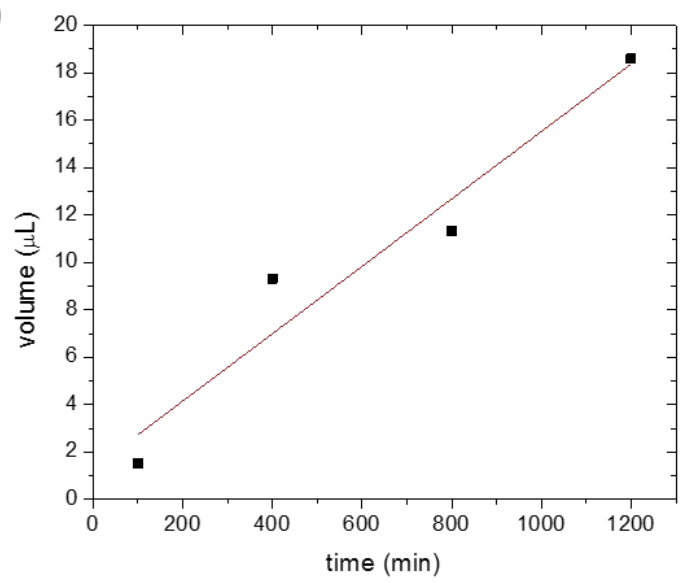

Figure S3. (a) Nematic height fraction in the capillary tube with distance along temperature gradient at different time. The fitting of each nematic height profile at each time is done with two lines, with the one near the hot end becoming flat due to meniscus effect. (b) The volume of nematic phase formed as a result of thermophoresis of nanoplate colloids as a function of time. Value at each time is obtained as area under the curves in part (a).

The volume of nematic phase at any given time is obtained as, $v_{N}(t)=W H \int_{x_{0}}^{x_{1}} f_{N}(x, t) d x$, where $x_{0}$ and $x_{1}$ represent the starting and the end point of nematic phase along the direction of the temperature gradient. Where $\mathrm{H}$ is the thickness of the capillary and $\mathrm{W}$ is its height which were $0.2 \mathrm{~mm}$ and $2 \mathrm{~mm}$ respectively. Supplementary video 1 is in compressed form which in real time represents 20hours of thermophoresis on isotropic sample of $\mathrm{ZrP}$ nanoplate colloids with $\phi=3.19 \%$, hot end (right) temperature $41^{\circ} \mathrm{C}$ and cold end 
temperature $28^{\circ} \mathrm{C}$. The sample length is $26 \mathrm{~mm}$ and height $2 \mathrm{~mm}$. The sample is kept between crossed polarizers.

We simulated the streamline of flow and the scale of the flow velocity driven by natural convection at steady state conditions. If nanoplates were considered as stable with uniform properties in the entire computational domain, then nanoplates and water can be considered as one phase. To simplify the calculation, properties of water were used in the simulation. The CFD simulation was performed using Ansys Fluent. The boundary conditions and geometries of the simulation domain are the same with the Eulerian approach. K- $\varepsilon$ model is used to simulate the flow. Figure S5 shows the contours of velocity magnitude. Two jet flows with opposite flowing directions exist in the domain. In the four corners of the domain, dead zones exist, where fluid flow velocity is almost zero. If nanoparticles flowing into the bottom dead zones, they can settle down and accumulate in these regions.

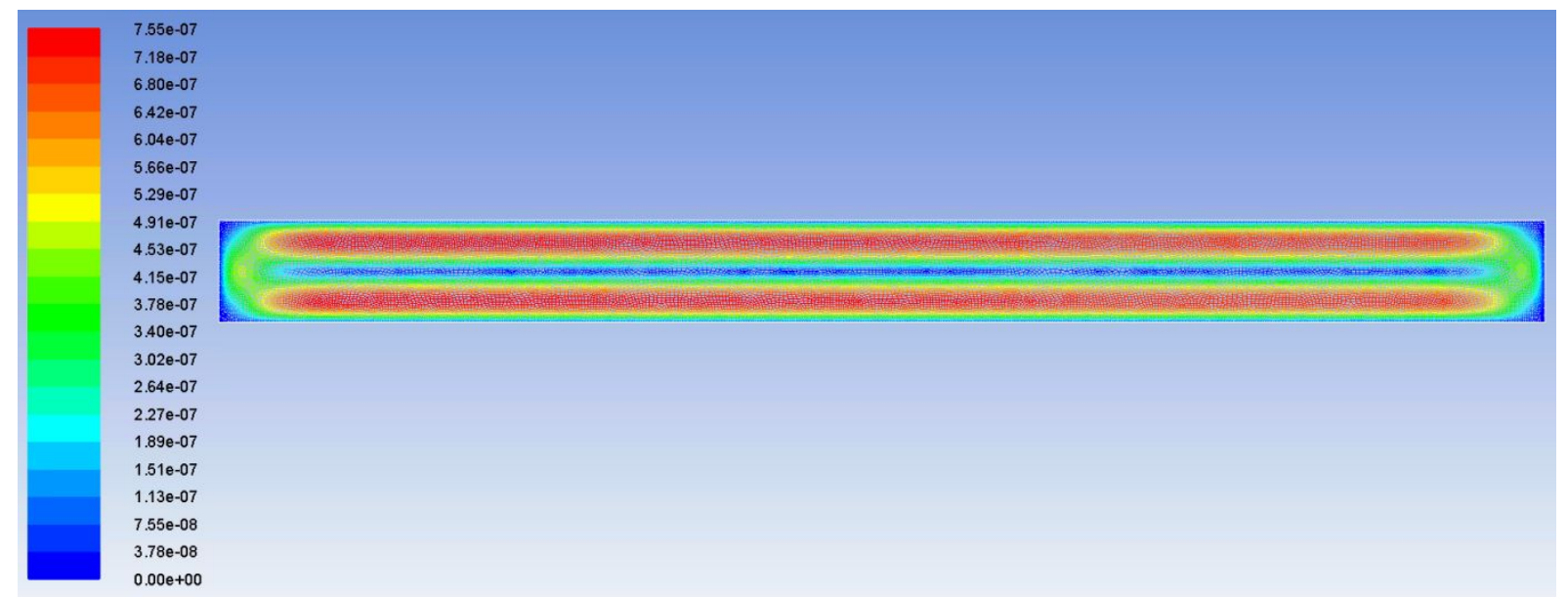

Figure S4. Velocity contour of the fluid within the enclosure under temperature gradient

We also estimated the scales of the horizontal and vertical flow velocity. If it is assumed that the fluid is single-component and that there is no internal heat generation in the fluid, the governing equation for internal convection can be written as follows.

$$
\begin{gathered}
\frac{\partial v_{x}}{\partial x}+\frac{\partial v_{y}}{\partial y}+\frac{\partial v_{z}}{\partial z}=0 \\
\frac{\partial v_{x}}{\partial t}+v_{x} \frac{\partial v_{x}}{\partial x}+v_{y} \frac{\partial v_{x}}{\partial y}+v_{z} \frac{\partial v_{x}}{\partial z}=-\frac{1 \partial P}{\rho \partial x}+\mathrm{v}\left(\frac{\partial^{2} v_{x}}{\partial x^{2}}+\frac{\partial^{2} v_{x}}{\partial y^{2}}+\frac{\partial^{2} v_{x}}{\partial z^{2}}\right) \\
\frac{\partial v_{y}}{\partial t}+v_{x} \frac{\partial v_{y}}{\partial x}+v_{y} \frac{\partial v_{y}}{\partial y}+v_{z} \frac{\partial v_{y}}{\partial z}=-\frac{1 \partial P}{\rho \partial y}+\mathrm{v}\left(\frac{\partial^{2} v_{y}}{\partial x^{2}}+\frac{\partial^{2} v_{y}}{\partial y^{2}}+\frac{\partial^{2} v_{y}}{\partial z^{2}}\right)-g[1-\beta \Delta T] \\
\frac{\partial v_{z}}{\partial t}+v_{x} \frac{\partial v_{z}}{\partial x}+v_{y} \frac{\partial v_{z}}{\partial y}+v_{z} \frac{\partial v_{z}}{\partial z}=-\frac{1 \partial P}{\rho \partial z}+\mathrm{v}\left(\frac{\partial^{2} v_{y}}{\partial x^{2}}+\frac{\partial^{2} v_{y}}{\partial y^{2}}+\frac{\partial^{2} v_{y}}{\partial z^{2}}\right) \\
\frac{\partial T}{\partial t}+v_{x} \frac{\partial T}{\partial x}+v_{y} \frac{\partial T}{\partial y}+v_{z} \frac{\partial T}{\partial z}=\alpha\left(\frac{\partial^{2} T}{\partial x^{2}}+\frac{\partial^{2} T}{\partial y^{2}}+\frac{\partial^{2} T}{\partial z^{2}}\right)
\end{gathered}
$$


To estimate the scale of the horizontal velocity in the top or bottom of the enclosure, one can combine equations (2) and (3) by eliminating the pressure to obtain:

$$
\begin{gathered}
\frac{\partial}{\partial x}\left(\frac{\partial v_{x}}{\partial t}+v_{x} \frac{\partial v_{x}}{\partial x}+v_{y} \frac{\partial v_{x}}{\partial y}+v_{z} \frac{\partial v_{x}}{\partial z}\right)-\frac{\partial}{\partial y}\left(\frac{\partial v_{y}}{\partial t}+v_{x} \frac{\partial v_{y}}{\partial x}+v_{y} \frac{\partial v_{y}}{\partial y}+v_{z} \frac{\partial v_{y}}{\partial z}\right) \\
=v\left[\frac{\partial}{\partial x}\left(\frac{\partial^{2} v_{y}}{\partial x^{2}}+\frac{\partial^{2} v_{y}}{\partial y^{2}}+\frac{\partial^{2} v_{y}}{\partial z^{2}}\right)-\frac{\partial}{\partial y}\left(\frac{\partial^{2} v_{x}}{\partial x^{2}}+\frac{\partial^{2} v_{x}}{\partial y^{2}}+\frac{\partial^{2} v_{x}}{\partial z^{2}}\right)\right]+g \beta \frac{\partial T}{\partial x}
\end{gathered}
$$

If steady state is reached, the transient terms, $\frac{\partial v_{x}}{\partial t}$ and $\frac{\partial v_{y}}{\partial t}$ should be ignored. Also, one can assume the relations: $\partial x \sim L=2.6 * 10^{-2}, \partial y \sim H=2 * 10^{-3}, \partial z \sim W=2 * 10^{-4}, \partial T \sim \Delta T, \partial v_{y} \sim v_{y}$, and $\partial v_{x} \sim v_{x}$. Since

$\frac{1}{\partial z^{2}} \gg \frac{1}{\partial z}>\frac{1}{\partial y}>\frac{1}{\partial x}$ and in the horizontal domain, $v_{x} \gg v_{y}$, eqn. (6) can be simplified as:

$$
0=\mathrm{v}\left[-\frac{1}{H}\left(\frac{v_{x}}{W^{2}}\right)\right]+g \beta \frac{\Delta T}{L}
$$

Then, horizontal velocity $v_{x}$ can be estimated as

$$
v_{x}=\frac{W^{2}}{\mathrm{v}} H g \beta \frac{\Delta T}{L}=\frac{\left(2 * 10^{-4}\right)^{2} * 2 * 10^{-3} * 9.8 * 2 * 10^{-4} * 14}{10^{-6} * 2.6 * 10^{-2}}=8.44 * 10^{-5} \mathrm{~m} / \mathrm{s}
$$

To estimate the scale of the vertical velocity in the left or right domain of the enclosure, one can also use eqn (6). Similar simplification can be used, except $v_{y} \gg v_{x}$, eqn. (6) can be simplified as:

$$
0=\mathrm{v}\left[-\left(\frac{v_{y}}{W^{2}}\right)\right]+g \beta \Delta T
$$

Then, horizontal velocity $v_{y}$ can be estimated as

$$
v_{y}=\frac{W^{2}}{\mathrm{v}} g \beta \Delta T=\frac{\left(2 * 10^{-4}\right)^{2} * 9.8 * 2 * 10^{-4} * 14}{10^{-6}}=1.1 * 10^{-3} \mathrm{~m} / \mathrm{s}
$$

\section{Order of magnitude estimate of Soret coefficient}


This section describes an approximate calculation of Soret coefficient of nanoplates. Following Figure 4, in the triangular isotropic region up to height $\mathrm{H} / 4$ from the bottom, the convection is negligible and a net positive density gradient a density gradient in the direction perpendicular to the I-N interface can be assumed to be linear. Concentration of the isotropic phase in the top $3 / 4^{\text {th }}$ section was uniform at $\phi_{1}$ due to persistent convection.

$$
\phi(l)=a l+b ; \text { for } 0 \leq l \leq l_{1}
$$

Using the boundary conditions of nanoplate concentrations:

$$
\begin{aligned}
& \phi(0)=\phi_{1} \\
& \phi\left(l_{1}\right)=\phi_{\mathrm{I}} \quad \text { where } l_{1}=\frac{H}{4} \cos \theta ;
\end{aligned}
$$

Thus we get,

$$
\phi(l)=\frac{\left(\phi_{\mathrm{I}}-\phi_{1}\right)}{l_{1}} l+\phi_{1} \quad ; \text { for } 0 \leq l \leq l_{1}
$$

By performing a balance between the initial number of nanoplates before applying temperature gradient and that at steady state, the concentration gradient along $l$ can be obtained.

$$
\phi_{o} L H W=\phi_{1}\left(H-\frac{H}{4}\right) L W+W \int_{0}^{l_{1}} \phi(l) p_{1}(l) d l+\frac{\phi_{N}}{2}\left(L-\frac{L_{1}}{2}\right) W
$$

where $p_{1}(l)=2 l / \sin 2 \theta$ is the length of an elemental section of the integration in the isotropic triangle in the bottom $1 / 4^{\text {th }}$ section of the sample (please refer Figure 3)

Solving we get,

$$
\begin{gathered}
\frac{\partial \phi}{\partial l}=a=0.037^{\circ} \mathrm{K} / \mathrm{mm} \\
\phi_{1}=0.023 \\
\sin \theta \frac{\partial \phi}{\partial l}=\frac{\partial \phi}{\partial x} \\
S_{T}=-\frac{\partial \phi}{\phi \frac{\partial T}{\partial x}}=-\frac{1 \frac{\partial \phi}{\partial x}}{\frac{\partial \partial T}{\partial x}}=-0.093 /{ }^{\circ} \mathrm{K}
\end{gathered}
$$

\section{Effect of temperature on nanoplate stability}

It is possible that some of the $\mathrm{Bu}_{4} \mathrm{~N}^{+}(\mathrm{TBA}+)$ ions may disengage from zirconium phosphate nanoplate at higher temperatures. If that happens, electrostatic repulsion between nanoplates would decrease and nanoplates would 
aggregate. In our experiment, no aggregates were observed to the best of our knowledge. To further confirm that no such disengagement of TBA+ ions took place, we performed zeta potential measurement of zirconium phosphate nanoplates at different temperatures as shown in Figure S6. The error margins indicate polydisperse nature of the nanoplate sample. Mean values of Zeta potential remained relatively unchanged in the concerned temperature range and the value was $-30 \mathrm{mV}$ even at temperatures around $40-50^{\circ} \mathrm{C}$ indicating negligible disengagement of $\mathrm{TBA}+$ ions from nanoplates.

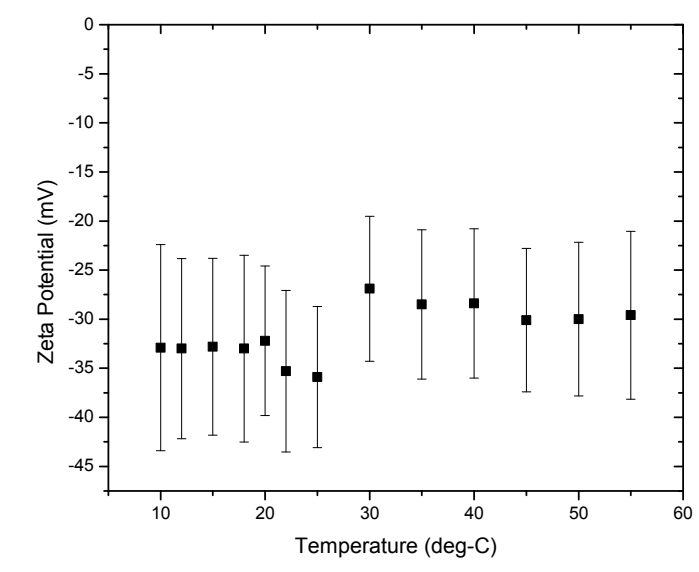

Figure S5. Temperature dependence of zeta potential of zirconium phosphate nanoplate

Structure of the nematic phase formed in presence of temperature gradient

(a)
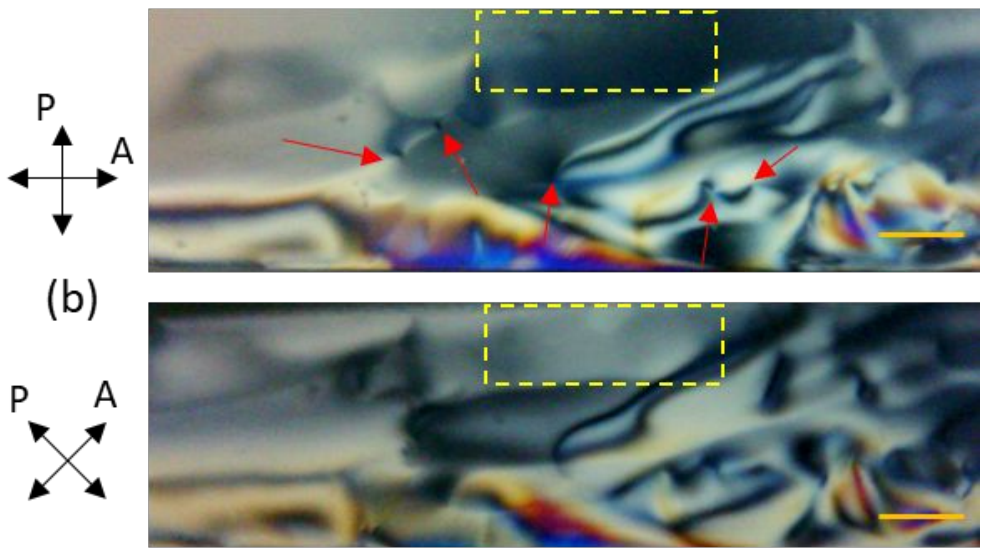

(c)

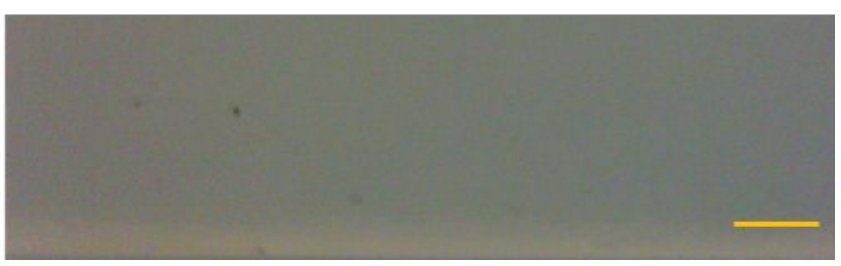

Figure S6. Texture of nematic phase developed due to temperature gradient driven growth. (a) Crossed polarized image with polarizers along vertical and horizontal direction. Characteristic $\pm 1 / 2$ defects of the phase are indicated by red arrows. Region outlined in yellow indicated one of the large 
nematic domains (b) Crossed polarized image with polarizers making at $45^{\circ}$ with vertical direction. Notice that the outlined turned from dark to bright indicating orientation of nematic director along wither horizontal or vertical direction. (c) Bright field image of the same region. Scale bar: $250 \mu \mathrm{m}$ 